\title{
BMJ Open Ten years of NIHR research training: perceptions of the programmes: a qualitative interview study
}

\author{
Paula Burkinshaw, ${ }^{1}$ Louise D Bryant (D) , ${ }^{2}$ Caroline Magee (D) , ${ }^{3}$ Peter Thompson, ${ }^{3}$ \\ Lisa Ann Cotterill (D), ${ }^{3}$ Matthew R Mulvey (D) , ${ }^{2}$ Jenny Hewison ${ }^{2}$
}

To cite: Burkinshaw $P$, Bryant LD, Magee C, et al. Ten years of NIHR research training: perceptions of the programmes: a qualitative interview study. BMJ Open 2022;12:e046410. doi:10.1136/ bmjopen-2020-046410

- Prepublication history for this paper is available online. To view these files, please visit the journal online (http://dx.doi. org/10.1136/bmjopen-2020046410).

Received 15 December 2020 Accepted 25 November 2021

\section{SLinked}

- http://dx.doi.org/10.1136/ bmjopen-2020-046368

Check for updates

(C) Author(s) (or their employer(s)) 2022. Re-use permitted under CC BY-NC. No commercial re-use. See rights and permissions. Published by BMJ.

${ }^{1}$ Leeds University Business School, University of Leeds, Leeds, UK

${ }^{2}$ Leeds Institute of Health Sciences, University of Leeds, Leeds, UK

${ }^{3}$ Executive Team, NIHR Academy, Leeds, UK

Correspondence to Dr Matthew R Mulvey; M.R.Mulvey@leeds.ac.uk

\section{ABSTRACT}

Objectives The UK National Institute for Health Research (NIHR) training programmes were created to build and sustain research capacity in healthcare. Following the training programme 10-year strategic review, this qualitative study aimed to deepen understanding of facilitators and barriers for those progressing through NIHR-supported research careers.

Design Semistructured qualitative study.

Data collection and analysis Telephone interviews conducted between May and August 2017 were digitally recorded, transcribed and analysed using Framework Approach.

Setting UK National Health Service (NHS) Trusts, university medical schools, District General Hospitals, Integrated Academic Training Programme centres and Research Design Services across the North East, North West, South East and South West of England, London and the Midlands.

Participants Fourteen women and eight men, of whom, 14 were previous or current NIHR personal awardees (seven doctors and seven allied health professionals (AHPs) or nurses) and eight were managers (staff within clinical or university training-related roles).

Results (1) NIHR awards were viewed as transformative for research careers; (2) however, there were perceptions of a biased 'playing field'. (3) Inequalities were perceived for AHPs and nurses, those outside of established research institutes and those in 'unfashionable' specialisms. (4) While support for NIHR awards contributed to a healthy research culture, (5) short-term awards were perceived as a barrier to continuing an independent research career. Conclusions Participants perceived many strengths of the NIHR training programmes in terms of developing individual careers and research capacity. Areas in which improvement could enhance the ability to attract, develop and retain researcher were identified. Our findings are of relevance to schemes in other countries, where healthcare researchers experience similar challenges. Further work is needed to overcome barriers and ensure equity of access to, and success within, clinical research training schemes to sustain the research workforce needed to address future global health challenges.

\section{INTRODUCTION}

The necessity of developing and sustaining a strong, global clinical academic workforce

\section{Strengths and limitations of this study}

- Interviews by a researcher independent of the National Institute for Health Research (NIHR) enabled personal awardees and managers to speak freely about their experiences and perceptions.

- Interviews provided rich data to illustrate findings from the quantitative review and identify perceived strengths and limitations of the programmes.

- Participants were selected from a diverse range of geographical locations, specialties, professions and host organisations.

- Findings have underpinned the NIHR review, which has already led to changes by the NIHR to address key recommendations.

- As a qualitative study with a relatively small sample size for a population with complex intersections between programme, role and demographic, the findings may not be generalisable to the wider population of NIHR programme personal awardees and managers or awardees of other such schemes internationally.

has been evidenced by the speed with which this workforce has responded to the multiple challenges of the COVID-19 pandemic. ${ }^{1}$ In the UK, an overall decline in numbers of clinical academics has been identified, and the need for action to secure the future of a clinical academic workforce is a national priority. ${ }^{2}$ This concern is shared by a number of countries worldwide, particularly in relation to delivering the research capacity needs of the future, but there is also agreement that a satisfactory solution to the problem has not yet been found. ${ }^{13-5} \mathrm{~A}$ number of studies have used qualitative approaches to examine factors encouraging or deterring clinical professionals from pursuing an academic career. Even in medicine, where research is widely valued and appointments combining clinical and academic roles are well established, difficulties in reconciling different role demands are common and a research career can still be seen as insecure 

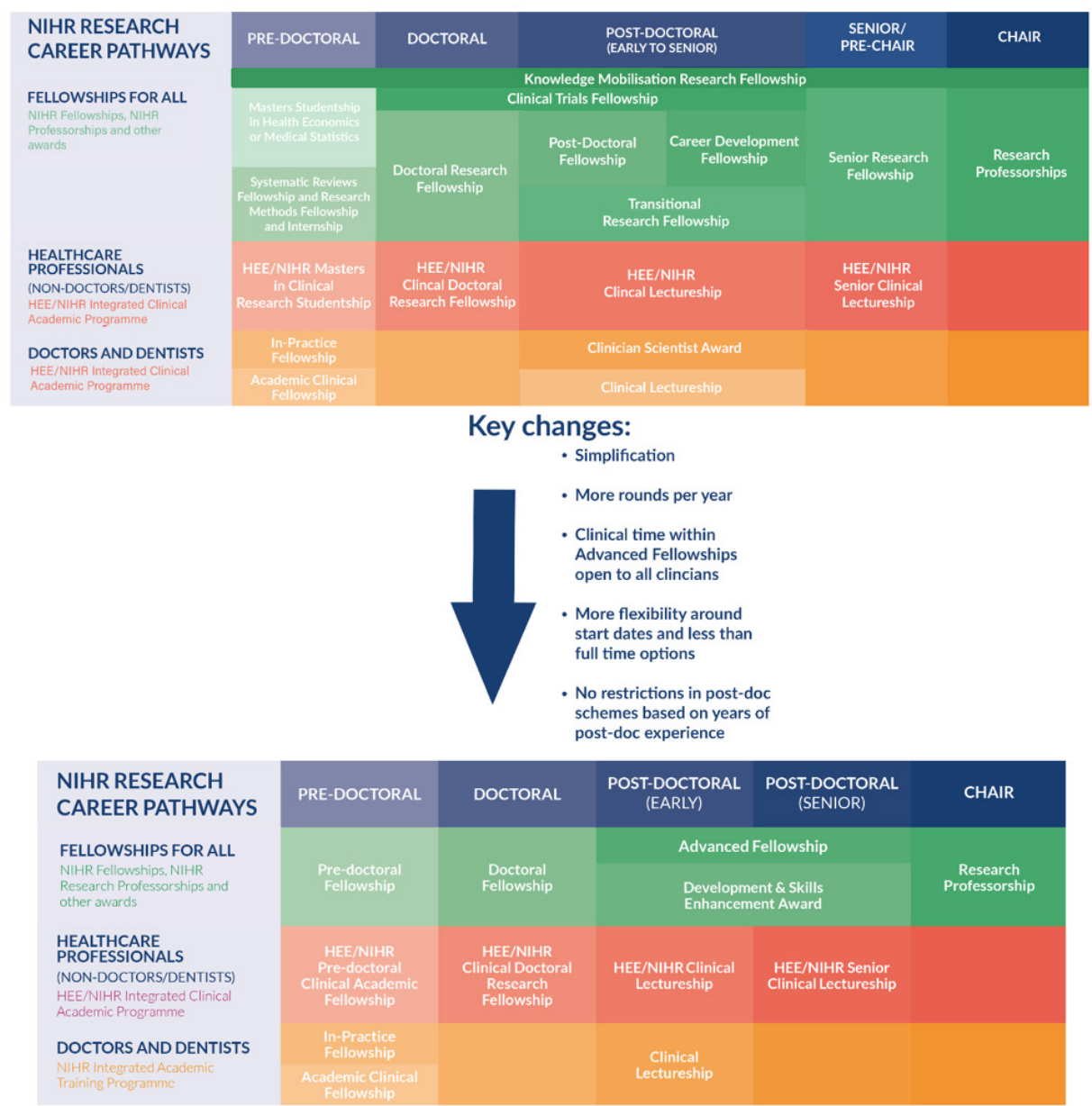

Figure 1 Key changes made in 2017/2018. HEE, higher education establishments; NIHR, National Institute of Health Research.

and unattractive. ${ }^{6-10}$ In the non-medical clinical professions, there is evidence of additional barriers: clinical managers who do not value research expertise, limited infrastructure support and an absence of research career pathways beyond doctoral level-all factors potentially amplifying more widespread gender inequalities between the professions. ${ }^{11-14}$

In the UK, the National Institute for Health Research (NIHR) Academy (formerly known as the Trainees Co-ordinating Centre) was set up in 2006 to help build national research capacity in healthcare by supporting research leaders of the future. ${ }^{15}$ A range of schemes supported professionals across medicine, dentistry, nursing, midwifery, the allied health professions (AHPs) and other clinical and non-clinical professions from predoctoral level to professor level (see figure 1). Recognising that new priorities in research, health and care had emerged since the schemes were first established; in 2016, the NIHR commissioned a 10-year retrospective Strategic Training Review to 'review the past and look proactively at future training needs'. ${ }^{16}$ The review, which was conducted by NIHR in collaboration with researchers at the University of Leeds, had a quantitative and a qualitative component. The quantitative component provided an analysis of application success (ie, funded or not funded) using existing data routinely collected by the NIHR Academy on training awards across funding schemes. The findings which were summarised in the 2017 report 'Ten years on: adapting and evolving to new challenges in developing tomorrow's health research leaders ${ }^{15}$ were largely positive, but success rates were found to vary by profession, and there was some evidence of attrition for women after the postdoctoral level. The purpose of the qualitative component was to deepen understanding of factors that may act as barriers and facilitators for those progressing through NIHR research careers and so complement the quantitative analysis being conducted in parallel, and allowing the NIHR Academy to introduce the right interventions to improve career pathways and career progression. Understanding where any inequalities in allocation of funding across disciplines or institutions lay was a focus of the review, and it was anticipated that in-depth interviews with individuals from different professions may inform this understanding.

We used Framework Approach, a rigorous applied qualitative methodology, developed specifically for policyrelevant research, in which the objectives and research questions are typically set in advance and shaped by the 
information requirements of the funding body. ${ }^{17} 18$ The interviews were designed to answer questions directly relevant to the aims of the NIHR Academy strategic review, that is to inform future policies and practice. Our aim was to identify areas where the NIHR Academy was doing well in relation to trainee experiences and where improvements could be made. The questions were agreed in collaboration with the chief investigator for both the qualitative and quantitative components $(\mathrm{JH})$.

\section{METHODS}

\section{Design}

Single, in-depth structured interviews with interview schedules developed in collaboration with the Academy to address key aims of the review. Interview schedules were piloted with two personal awardees and one manager.

\section{Sample and recruitment}

A purposive sampling framework aimed at recruiting 16 NIHR personal awardees employed a $2 \times 2$ table (gender (female/male) by professional role (medically qualified/ AHP or nurse)). Medically qualified staff make up the largest proportion of applicants, and the NIHR Academy was concerned that there appeared to be lower success rates (ie, funded personal awards) for non-medically qualified applicants, particularly nurses. ${ }^{15}$ Limiting the number of roles also enabled sampling for diversity within roles and well as across roles, in terms of seniority and geography. We also aimed to recruit eight staff within clinical or university roles who had expertise in capacity development or the support of NIHR awardees. Purposive sampling was used to ensure diversity of participant across scheme, geographical location and host institution type (university, National Health Service (NHS) trust, other). Using these criteria, the NIHR Academy approached current or previous personal awardees and research managers by email or telephone. Only individuals who had ultimately been successful in application were able to be participants as the NIHR Academy only had consent to contact personal awardees.

Protecting the identity and privacy of participants was paramount and several steps were taken to ensure this.

1. The NIHR Academy asked identified individuals for permission for their contact details to be passed on the lead researcher $(\mathrm{PB})$.

2. The research supervisors at the University of Leeds (LDB, JH) were not privy to the details of potential or actual participants at any point.

3. The lead researcher who conducted the interviews was independent of the NIHR Academy and the University department from where the study was run.

4. The interviews were transcribed by an approved independent professional transcription service covered by The Data Protection Act. Transcripts were checked for accuracy by the lead researcher.

5. Transcripts were anonymised by the lead researcher before they were accessible to the research supervisors.
6. Participants gave explicit permission to use anonymised quotes as part of the informed consent process and no direct quotation or equivalent is associated with information that potentially may be linked to any participant.

The lead researcher was an experienced interviewer and qualitative researcher with expertise and interests in the area of gender inequality in higher education. She contacted individuals identified by the NIHR Academy to inform them of the study, invite them to participate and set a telephone interview time if appropriate. Seventeen individuals declined or did not respond after follow-up. Consent to contact details were not forwarded for one person who was approached by the NIHR Academy, and the researcher was ultimately unable to get in contact with one person who had initially agreed to be interviewed. Telephone interviews were conducted in a setting chosen by participants (home or work) and digitally recorded with the consent of the participant. On average, the interviews lasted around $45 \mathrm{~min}$ with a few more than an hour and the shortest less than half an hour.

\section{Patient and public involvement}

There was no patient or public involvement.

\section{Setting}

Broad geographical coverage within England was achieved with participants from the North East, North West, South East and South West of England, London and the Midlands. Participants worked within NHS Trusts, university medical schools, District General Hospitals (DGHs), Integrated Academic Training (IAT) Programme centres and Research Design Centres.

\section{Analysis}

Transcripts were imported into NVivo, a qualitative data analysis software package. ${ }^{19}$ The individual transcripts were analysed using the five stages of the Framework Approach. The approach uses a structured and rigorous approach to organising and thematically analysing interview data directly in relation to defined research questions. ${ }^{17}{ }^{18}$ The lead researcher undertook initial familiarisation with the data via immersion in the transcripts, which was followed by identifying an initial thematic framework based on the questions in the topic guide (see table 1). The analysis framework was developed by the researcher in consultation with the research supervisors and to address the two main research questions

For this reason, only aspects of the transcripts that helped answer these questions were coded. Each transcript was indexed, that is, annotated using codes based on the thematic framework. Once all transcripts were indexed, individual responses to questions were organised into charts by theme to give an overview to support data synthesis. The lead researcher conducted the indexing. Initial index codes, then possible and emerging themes within and across the charts, were discussed with LDB, who is experienced in using the Framework Approach. The lead researcher, LDB and JH—also an experienced supervisor of qualitative research-conducted a 
Table 1 Topic guide for personal awardees

\begin{tabular}{|c|c|}
\hline Question & Prompts \\
\hline $\begin{array}{l}\text { Can you tell me about your experience of the NIHR } \\
\text { training programmes? }\end{array}$ & $\begin{array}{l}\text { Which scheme did you apply for? } \\
\text { When did you apply? } \\
\text { How did you decide which scheme to apply for? }\end{array}$ \\
\hline $\begin{array}{l}\text { What kind of support did you receive from your } \\
\text { institution in applying? }\end{array}$ & $\begin{array}{l}\text { What did you find helpful in the application process? } \\
\text { What did you find unhelpful } \\
\text { Do you think your Institution provides sufficient support to } \\
\text { researchers in your field in terms of applying for the NIHR training } \\
\text { programmes? } \\
\text { If so what support do they provide? } \\
\text { If not what support could they provide? }\end{array}$ \\
\hline $\begin{array}{l}\text { Are the NIHR training programmes currently attractive } \\
\text { to researchers in your field? }\end{array}$ & $\begin{array}{l}\text { Does your Institution have role models - that is, senior staff with a } \\
\text { track record of NIHR programme awards in your research field? } \\
\text { What attracted you to the NIHR training programmes? } \\
\text { Do you think some people are put off from applying? If so .... why? } \\
\text { Could the NIHR academy do anything about this? }\end{array}$ \\
\hline If you were unsuccessful...... & $\begin{array}{l}\text { What has been the impact of being unsuccessful on your career } \\
\text { progression? }\end{array}$ \\
\hline $\begin{array}{l}\text { Would you consider applying for another NIHR training } \\
\text { programme? }\end{array}$ & $\begin{array}{l}\text { If so why? } \\
\text { If not why not? }\end{array}$ \\
\hline Would you encourage a colleague to apply? & $\begin{array}{l}\text { If so, why? } \\
\text { If not why not? }\end{array}$ \\
\hline
\end{tabular}

Is there anything else you would like to tell us about your experience of the NIHR training programmes?

NIHR, National Institute of Health Research.

mapping and interpretation exercise, to use themes to address the research questions. Any differences in interpretation were discussed at length during project meetings until consensus was achieved.

\section{Findings}

We interviewed 22 individuals: 14 women and 8 men. Of the 22, 14 were personal awardees (previous or current) and 8 were managers. Personal awardees included seven doctors and seven AHPss or nurses. Participants had experience of a range of NIHR funding schemes between them (see figure 1 for NIHR funding schemes). A number of the personal awardees had applied unsuccessfully previously, broadening the range of experience accessed.

The themes are presented in a narrative structure in relation to the questions in figure 2. The themes highlighted a number of perceptions; some of these were not borne out in the evidence collected through analysis of NIHR Academy programme data, ${ }^{20}$ and this is addressed in the Discussion section.

\section{Theme 1: NIHR awards as transformative}

NIHR awards were perceived as of high value and 'transformative'.

The award I think is transformational [for] my career progression, on my ideas for what my career could be, ...confidence that I can achieve things on a much bigger scale and with potentially greater impact than I would have otherwise thought (nurse/AHP).

I think it has quite a profound impact, really. So, if I hadn't had done this scheme, I think I probably would have remained in clinical practice for the rest of my career (nurse/ $\mathrm{AHP}$ ).

Well, I always wanted to have my own group, but I think this has meant it was actually possible to do that rather than it being a pipe dream (medical doctor).

The fellowship has altered my career trajectory in that if I had not had the fellowship, I would not have any prospect of having an academic career (medical doctor).

Personal awardees identify positively with the NIHR and can feel part of the wider organisation:

We encourage people to apply because of the additional benefits that they're probably not aware of, but ..., being part of the NIHR family and the access to all of those resources and access to the training programme that comes with that award (manager).

\section{The value of competitiveness}

Managers and personal awardees mainly agreed that the competition associated with the awards was a critical feature of their prestige and attraction. 


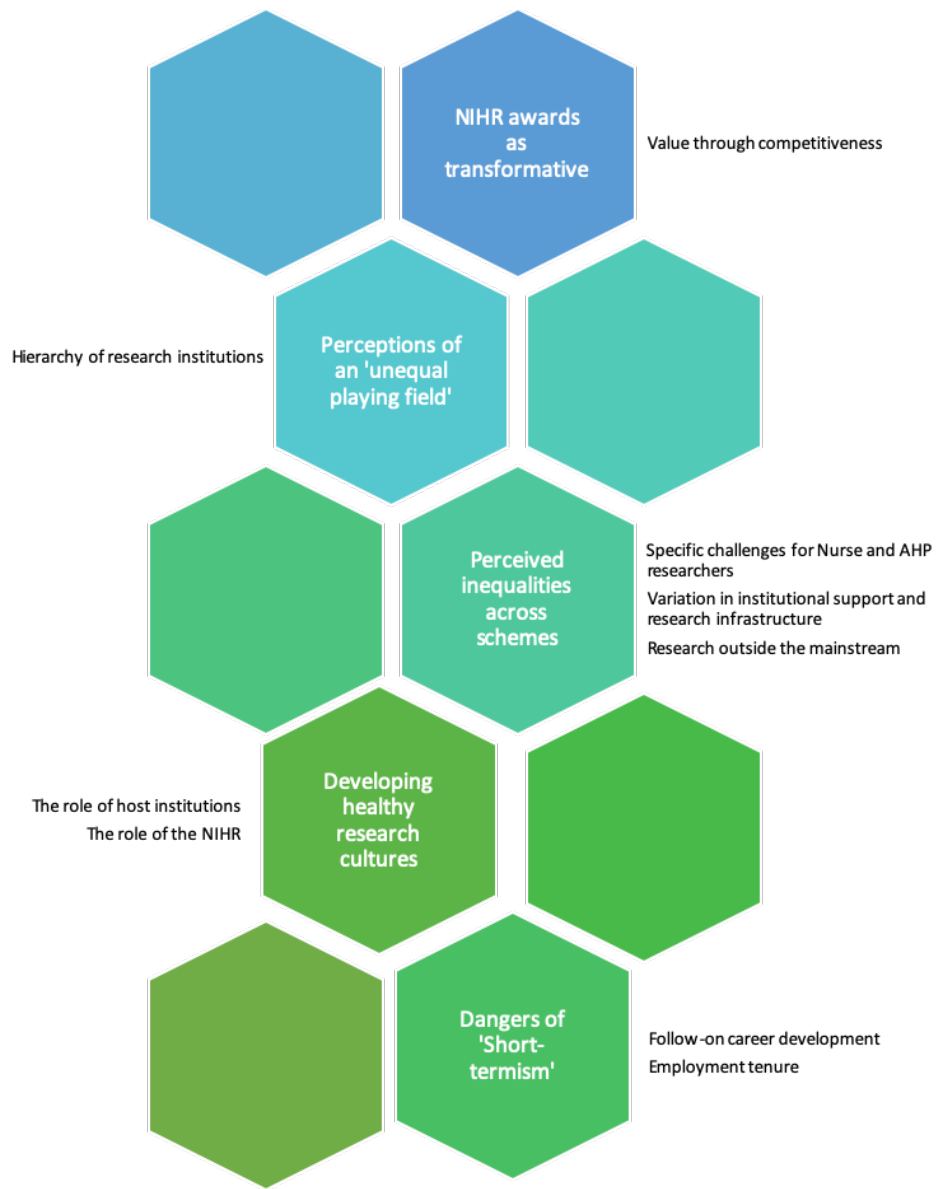

Figure 2 Main and subthemes identified within the interviews. AHP, allied health professional; NIHR, National Institute of Health Research.

It's also the kudos, so people like to apply to be an NIHR ACF (Academic Clinical Fellow) (manager).

It's also that external validity, somebody else outside your institution has appraised what you're doing and thinks it's of an appropriate standard. No matter where you are that is a good thing (medical doctor).

\section{Theme 2: an unequal 'playing field'}

Personal awardees who were medically qualified and managers were least likely to see a problem with the current system; however, some interviewees felt that the success of applicants, as judged by the panel, depended a great deal on the size and reputation of their institution.

\section{A privileged 'in club'}

For some, there was a perception that larger and 'Golden Triangle' UK institutions (Oxford, Cambridge and some London Universities) fared best in terms of success, and that an application from 'the wrong sort of Trust linked to the wrong sort of universi$t y$ ' [Manager] would not succeed.

One of the criticisms I hear of NIHR is, you know, if you're not in the club.... you won't get the funding ... they need to be able to help people who might be excellent but in a different institute for all sorts of reasons (nurse/AHP).
I think it's more difficult for some of the DGH's where that NIHR money doesn't tend to flow (manager).

Some managers and personal awardees perceived a 'lack of transparency' around how decisions are being made by the NIHR, with a hierarchy of (host) institutions topped by those 'in the club'. In this view, application review panels are perceived to be made up of members from the club' with privileged 'insider knowledge' that is beneficial to their own institution's applications. Hence, the award system was seen as being in in danger of becoming a 'closed shop' (manager), which some participants consider 'unhealthy' and 'elitist' (manager).

So I mean a review of all of the grant-giving bodies to ensure fairness and equity across all of the institutions, to ensure that they're not stuffed full of people from the 'Golden Triangle', might be quite helpful (manager).

\section{Theme 3: perceived inequalities across schemes}

A number of subthemes were identified underneath an overarching theme of perceived inequalities in relation to funding outcomes within or across schemes.

Specific challenges for nurse and AHP researchers

A common subtheme emerged around the perception that fewer awards were made to nurses/AHPs than 
medical doctors. It was felt that the working patterns of nurses and midwifes disadvantage applications for awards because research is not embedded, encouraged or valued in nurse/AHP culture to the same extent as for doctors.

'Whereas if a nurse especially, or an AHP says it [I'm doing research], it's still a bit of 'ah oh', sort of reaction you get...... people look at it and say, "Oh well, it's not real research is it?" When you're not a medic. Really frustrating... All the people I know who've got things like a fellowship it's because they've gone out there and found it themselves. It's not so much because someone said, "Are you aware this is available for you?" (nurse/AHP).

Another perception was that nurses/AHPs tend to be more experienced when they do embark on research leadership, meaning they do not fit as easily into the standard research career trajectory.

What tends to happen is people go into jobs ... and specialise ... they're getting to the point where they're saying, "Actually there's all these questions that I have not answered, I now need to go and do some research." At that point often they're too senior really to fit in nicely with the NIHR pathway. But no one has identified that as an issue (nurse/AHP).

The nurses/AHPs in our study believed that while the research they did was often undervalued by their host institution, they perceived that a sustainable future for the NHS will depend on evidence-based nursing and AHP practice.

... a lot of what we do as nurses doesn't have much of an evidence base. ... There are probably lots of better ways to do things that could potentially save money for the NHS and improve the patient experience...... if you talk to most nurses they've got some great research ideas but they've got no idea of how to actually convert that into a research study and tackle it (nurse/AHP).

There was a view that the requirement for candidates to demonstrate research experience, as evidenced by publications, disadvantage nurse/AHP applicants, in particular, and those who have fewer opportunities to do research. Managers felt strongly that readiness to embark on research leadership could be evidenced in other ways. They felt that individuals whose institution or role allows them no opportunity for independent research, grant applications or entrepreneurial awareness find this requirement a barrier to success.

I anticipated that it would be harder to get [than for medical doctors] and that I would find it harder to get because of things like I didn't have enough publications and yeah, I didn't get it (nurse/AHP).

No explicit references to gender inequality within the NIHR Academy schemes were made by any participant. Most personal awardees said they were motivated to some extent by role models in their field. They themselves became role models later on and appreciated the value and responsibility of being so. Some women participants remarked on a need for a greater diversity of female case studies and NIHR ambassadors to provide a wider range of role models.

We do have some of the NIHR 'poster girls' and ours tend to be nurses and AHPs. We don't really have any medics (manager).

Some participants would have liked greater promotion of part time opportunities, and successful exemplars, of those (mostly women) who work in this way. References were made to 'other commitments' (family responsibilities, a mortgage), which impact on career development at an earlier stage.

You're not aware of these fellowships until you're quite late on in your career. .... When you're older and you've got other commitments it's actually quite difficult. Then to get from a PhD to the next stage of fellowship, and the next stage.... it's quite a few years. So you probably need to start earlier (nurse/AHP).

Several NHS-hosted applicants said their institutions provided no dedicated time within work hours to complete applications. Applicants, therefore said they had to do everything in their evenings and weekends.

To write a successful fellowship application takes a lot of time and there's no kind of allowance for that, I had to do all of that in my own time... (nurse/AHP).

The onus being solely on the applicant to find that time and taking the hit in terms of weekends and evenings (nurse/ AHP).

Variation in institutional support and research infrastructure A second significant subtheme was the perceptions around inequalities in levels of institutional support for research and its perceived link to success-or lack of. In particular, there were perceptions that good practice developed by some universities can disadvantage applicants from elsewhere. Some common features of institutional infrastructure were seen to increase the likelihood of successful applications and a positive 'award life' experience. In the UK, universities that are part of the Russell Group (a group of research-intensive UK universities) have established systems and networks for supporting researchers, including those applying for NIHR awards. A good example is the routine reviewing and filtering of 'fledgling' applications before they progress to the next steps, culminating in a repository of grants/awards for future reference.

Where I am ... you announce your intention to apply for a Fellowship through a short form. They will then give you feedback on ... their view of what your likelihood of success is ..... Once that has happened and you have got the idea that the university is probably behind you, that gives the increased impetus to what you are doing (nurse/AHP).

Universities hosting an IAT were seen to enjoy greater freedom in terms of a devolved distribution of awards and 
being able to identify and prepare promising students and early career medical doctors to apply for awards that they administrate.

Well, because we bang on and on about them. Anybody [medical students] who looks as though they're vaguely academic, we have an INSPIRE programme, the AMS (Academy of Medical Sciences)/Wellcome scheme. We have an academic foundation programme, which I set up here a long time ago which, if you like, grooms them for academic careers (manager).

So there is a support scheme for people who are thinking of applying for an NIHR fellowship. They meet regularly and you have senior professors who run these support groups. So, they go through all the details, because the application is complex and you might need to know a lot of things, but there is very good support around NIHR funding (medical doctor).

They [IAT personal awardees] feel part of a separate school. They get good supervision. Their research time is protected. They love coming to the showcase events. They like the fact that the academic school is small enough that I know all of them individually (manager).

This can create a perception of an unequal playing field within institutions as interviewees within Schools of Healthcare, Nursing and Midwifery, for example, did not view themselves as benefitting from this kind of support. In the hospital setting, some perceived that research is 'not core business'.

We're not in a medical school-we haven't got a team of people who support people doing NIHR routes (manager).

As some NHS institutions do not prioritise award applications, there is a perception of little advice and guidance for applicants. In particular, help with navigating the forms, coordinating inputs from relevant people, negotiating the wider application process and the financial element, which was a 'minefield' for many. Applicants can be left feeling demoralised and undervalued, their time spent on unsuccessful applications viewed as a waste of investment rather than capacity building. Some spoke of the huge personal risk and 'burden' of taking on this speculative process (nurse/AHP).

Some personal awardees spoke of being 'fortunate' in having support from their line manager/supervisor and considered this support to have been critical to their success. The best managers are not only supportive during the application process but also encourage applicants beforehand to be ambitious for the future and to consider research leadership as a career:

I think well she's just a very good manager and she's very keen to sort of develop people in areas that they're interested in (nurse/AHP).

I had one key supervisor who was the head of the department which I was hoping to work in, who was enthusiastic about me applying and had a colleague who was also keen on me applying. They were the enthusiasts who encouraged me to apply and helped me develop my application (medical doctor).

This personal support from someone who values research is especially valuable for NHS applicants; individuals who will encourage you to apply, then support you through difficulties with NHS management. Not all personal awardees reported receiving this support.

Management is a completely different matter. Whether that's because I'm a nurse rather than a medic and I'm viewed differently, I don't know. But the NHS management is a very, very difficult thing to work with (nurse/AHP).

Those in NHS institutions often tended to rely on their partnering university for support. It is, therefore, unsurprising that nurses/AHPs and managers reported being concerned about the nature of the NHS host institution's relationship with their partnering universities. To them, it is critical for this relationship to work well:

I think that a lot of the NHS organisations... don't realise that income is at stake for the university. Often the university and the NHS have their own set of incentives and disincentives and they operate in different ways. So I do think that there is ... a sort of political issue there around who hosts the award, which might end up feeding into how much support you get (nurse/AHP).

Some interviewees felt the NIHR Academy could do more to encourage DGHs, for example, through institutional mentors/being partnered with more experienced and successful institutions.

My impression is [another institution] have people at all levels of NIHR awards from internships up to professors. So that is amazing and I think that they're one of the sort of beacon sites in England .... there is more support, there are just more role models for what's possible really and more people to support you to make something happen (nurse/ AHP).

\section{Research outside the mainstream}

A third subtheme was that some interviewees perceived inequalities by research specialism-speech therapy, mental health, general practice, surgery and paediatrics were all mentioned as fields, which are seen to be undervalued or were 'not trendy' (medical doctor).

I think community and mental health trusts generally are disadvantaged with the NIHR. I think most of the money and infrastructure and the training schemes go to the teaching hospitals (manager).

For the specialities where research is not embedded, there was a view that the NIHR Academy should encourage critical mass via research leadership support to create a track record of success to 'breed' more success. 


\section{Theme 4: developing healthy research cultures}

The role of the host institution

Cultural messages about the value of research in a host institution influence whether or not potential applicants' feel they have a possibility of an independent research career in their role or organisation. These messages are transmitted via numerous means, and many positive examples were provided by the interviewees: seminars to help people decide whether to apply for NIHR awards, talks from successful applicants about their experience and the process; workshops for preparing applications; mock panels and feedback sessions, research-focused training needs and analysis, award showcase events, research built into careers days for students, student research societies, award holder forums, internal research awards and internships, buddying with a successful applicant and postaward development and support, including networking and away days.

I found myself probably in the right place at the right time at the right hospital where there was an ethos of applying for these things (medical doctor).

So what we do there is we start with the medical students as soon as they come into medical school and we take them on a laboratory tour, we talk to them about the research that we get up to. We run an INSPIRE Programme, which is broken down into various groupings in various sub-specialties, where the medical students can get together with the senior academics and see the research that the senior academics are involved in, and get involved in research (manager).

These types of initiatives-coupled with visibility of role models and 'talent spotting' across a wide range of individuals-demonstrates a healthy research culture in those host institutions with a track record of successful applications.

Myself and a team of mentors, we will do some talent spotting and then pick people up and have a discussion with them about their career trajectory and we might suggest that they-if they're interested in research, we might suggest that they apply for the HEE internship or the master's or it may be the PhD; and we will support them in terms of identifying where they are and what their likelihood of success is (manager).

Not everyone experienced this level of support within their institution

It's quite clear that my field is kind of looked down upon by a lot of the senior members of the institution and [they] are very open about that (medical doctor).

\section{The role of the NIHR}

In order to level the 'playing field', a common suggestion was for the NIHR Academy to have more influence on the research leadership infrastructure of host institutions. Some interviewees considered the NIHR Academy to be 'too passive' in their expectations of host institutions, considering how prestigious these awards were considered to be and the powerful reputation of NIHR. Some interviewees referred to the requirement for institutions seeking funding for NIHR Biomedical Research Centres to hold Silver Athena Swan chartered status (this requirement was removed in 2020). The Athena Swan Charter is a framework used to recognise commitment to advancing gender equality in Higher Education in the UK, Ireland and Australia, where it is called the Science in Australia Gender Equity (SAGE) Athena Swan Charter. It was felt the NIHR had the power to insist on certain policies and practices being adopted by NIHR host institutions. Examples included improved commitment to employment tenure beyond the lifetime of an award, 'seamless' support structures between the NIHR and host institution support for some time out of day-to-day tasks to complete applications.

I think if NIHR made a point of saying that that was important to them then more would be done. I mean you just need to look at what's happened [with] Athena SWAN to see the impact that NIHR saying something's important can have on the way that a university behaves (nurse/AHP).

A number of interviewees called for greater clarification of the roles and responsibilities of supervisors and mentors and a greater consistency of experience for personal awardees.

NIHR have in effect these expectations of what the supervisory team and what the institution is going to be able to offer ... [however in some organisations] the infrastructure's weaker, or their supervisors,... haven't had NIHR, or haven't had big charity funded students (manager).

I think it would be really useful to have like some mentors... Our NIHR training locally, they have a ... sort ofinformal mentoring scheme where they kind of match you up to someone that's ahead of you, but there's no-one actually in my research area (medical doctor).

\section{Theme 5: 'short-termism'}

The final theme addressed the relatively short-term nature of the awards (ie, short-termism) with generally no expectations placed on institutions about further development or employment tenure. This can lead to award holders feeling they face a 'cliff edge' on completion (nurse/AHP).

So when people come back to practice, there's absolutely no time or capacity for them to write their papers, no obligation for the supervisors to continue to support that individual, so many people just get cut loose..... Unless they are embedded in a research group, there's also not the cultural emphasis there and, as yet, we don't have any clear career pathways (manager).

As nurses and AHPs tend to come to research later on in their careers their NHS salary band may be seen to be prohibitive for their institutions to follow through on promotions afterwards. 
When the employers are saying, "Well, okay, we could keep you on but you might have to go into a Band 5 job when you finish instead of your lovely Band 8"... . Whereas I think if you're a Band 5 or you're in a massive department, that's not an issue because there's leeway for people (nurse/ AHP).

They [AHPs] can get these fellowships but actually in terms of where their career progresses they hit the ceiling effect very quickly and I don't know how that's going to change within the NHS. They get to band 8 and there's nowhere for them to go. So what is the benefit of them having a PhD? (manager).

This situation may lead potential applicants to be 'risk averse' if they have seen evidence of the cliff edge in their institution.

The institution used to have to promise that they would employ you after the fellowship finished. Whereas now that is not the case. And so you do feel a little bit that you're vulnerable (medical doctor).

I just find it ironic that the whole NHS is supposed to be based on evidence-based practice, and yet trying to get support for researchers ... on permanent contracts and ... having to go from year to year wondering if you're going to have a job the next year (nurse/AHP).

Some talked about setting realistic expectations about the follow-on from an award and/or offering bridging funding for the next steps. Time and funding to publish papers or develop further grants appear rare and locally determined.

\section{DISCUSSION}

This interview study complements the findings in the 'Ten Years On' report and the quantitative analysis of the clinical personal award applications. The qualitative approach enabled greater understanding of some of the issues identified and brought to life the experiences and viewpoints of personal awardees and managers associated with NIHR awards previously supported through the Trainees Coordinating Centre (TCC). These insights may be of wider interest to those who lead on equivalent schemes in other parts of the world

Most of the literature on research careers has adopted a quantitative approach and has focused on medical professionals. Some studies have taken place in Australia, Canada and the UK, but the majority have been conducted in the USA. An interest in the careers of nonmedical professionals is a more recent development, as is the use of qualitative approaches, but these changes are mainly apparent in the Australian and UK literature. Vassie $e t$ al in the UK conducted a meta-thematic synthesis of factors identified from their scoping review of influences on medical research careers. ${ }^{9}$ Finn $e t$ al, also based in the UK, conducted a systematic review of the quantitative and the qualitative international literature on doctors' and dentists' research careers, supplemented by semistructured interviews with UK participants. ${ }^{8}$ In Australia, Matus et al conducted a systematic review of the international literature on research capacity building for AHPs. ${ }^{13}$ In addition to the interview data from the doctors and dentists in the Finn et $a l$ study, ${ }^{8}$ findings from primary qualitative research have been reported by Trusson et al in the UK (medical clinical academics, nurses, midwives and AHPs) ${ }^{14}$ Brandenberg and Ward in Australia (allied health clinicians) ${ }^{11}$ and Avery et al in the UK (nursing, midwifery and AHPs) ${ }^{21}$

In the sections that follow, the findings of the current study are placed in the context of this body of international work.

\section{Awards as transformative}

Our findings show that the awards are highly valued by individuals and the health research community; those who are awarded fellowships can view them as transformational. Our quantitative analysis of applications identified a twofold increase in the odds of an application being successful if the applicant had held a previous NIHR award, suggesting that early awards can be an advantage to those who want to pursue a career in health research. A mixed methods study assessing career progression of NIHR Academic Clinical Fellows also identified that awards can act as a 'springboard' to a research career. ${ }^{22}$

This finding is echoed by many reports in the literature, reflecting the experiences of people across the health professions and in a number of different countries. ${ }^{91314}$ Trusson et $\mathrm{ll}^{14}$ noted, however, that embarking on a clinical academic pathway could begin much earlier in medical professionals than in their non-medical counterparts, because the latter could not access a pathway in which clinical and academic training were deliberately and systematically combined.

\section{Perceptions of an 'unequal playing field'}

The findings in our companion paper suggest the NIHR Academy has succeeded in its objective of treating all applications equitably. ${ }^{20}$ Very large differences in numbers of awards to different professions are mainly the reflection of almost equally large differences in the numbers of applications. Nevertheless, we identified perceptions that the award system was an 'unequal playing field'. Greater transparency about the makeup of review panels is required to reassure applicants that the geographical spread of panellists is much broader than may be perceived. A perception that panellists gain 'insider information' to benefit applications from their own institutions further identifies the need for transparency in how awarding panels function in situations where a potential conflict of interest may arise.

The perception that some professions (medical doctors), specialities and elite institution groupings ('Golden Triangle' and 'Russell Group') are advantaged in comparison to others was not supported by our analysis, which showed that while applications from UK 'Golden Triangle' and 'Russell Group' constituted nearly 
$80 \%$ of the total over the assessment period, applicants, within these groupings, were not more successful than those from other institutions. ${ }^{20}$ Similarly, while applications from medical doctors account for more than a third of those submitted overall, once factors such as previous award are taken into consideration, professional background was only a significant predictor of success in specific and limited circumstances, and the differences found were not compatible with any kind of simple status hierarchy. ${ }^{20}$

One influential characteristic of the host institution did emerge from the analyses and that was if the institution had, or was associated with, a medical school. ${ }^{20}$ Our interviews suggest that a strong research infrastructure enables hosts to encourage and support a higher volume of award applications from an earlier stage. This is a factor particularly for doctors working within established medical schools where there has been a long history of enabling clinical academic careers. Such medical schools were already well situated to bring in and support NIHR schemes, and indeed the schemes were designed to work within existing structures. This cultural legacy may partly underpin the 'medical school effect', as medical schools are often colocated with good research infrastructure.

There were concerns about some 'unfashionable' specialities (speech therapy, mental health, general practice, surgery and paediatrics were mentioned), either in terms of lack of interest from host institutions or funding via the NIHR. These perceptions were not supported by our review, which identified that generic health, mental health and cancer are the three health categories with the most awards, and that paediatrics, general surgery and general psychiatry are well represented in IAT schemes. ${ }^{15}$ No in-depth analysis of success rates by specialty was performed in our quantitative study and inequalities in success rates by specialty may well exist; however, it is also possible that the small number of applications coming from some specialities may skew perceptions of associated success rates. The NIHR themselves have identified a need to build capacity in 'academically vulnerable' specialties such as intensive care and emergency medicine. ${ }^{15}$

Concern about unfashionable specialities has long been a feature of the literature on medical research careers. ${ }^{9}$ An analysis of awards from the Canadian Institutes of Health Research suggested a complex picture of interacting effects in which gender differences in success rates were much more apparent in some research areas (defined here by funder remit rather than applicant discipline) than others, leading the authors to call for the identification of 'bias-enhancing conditions in the peer-review process'. ${ }^{23}$ The present study lacked data on clinical specialty, but until such data are available, the lack of evidence of bias in respect of the gender, professional background or institutional prestige of applicants is reassuring. These findings do, however, serve to focus attention on the structural factors, also widely apparent in the literature, that support the research careers of some kinds of applicant much more than others.
Importance of a healthy research culture and institutional support

Many interviewees reported benefitting from a supportive infrastructure that embeds them in the research culture of their host institution and helps develop their research careers beyond the initial award. Managers working within these host institutions provided detail of how potential awardees are 'spotted' and supported within a system developed to bring awards to the host institution. In contrast, some personal awardees did not have access to a supportive institutional infrastructure. For some, mentoring or sponsorship appeared to rely on 'goodwill' or the support of enthusiastic colleagues. The main shortcoming in institutional support appeared most likely to affect (in this study) nurses and AHPs, those new to research yet senior in their clinical careers, or those based in small regional centres or DGHs without an established research culture. While the parallel quantitative analysis of applications did not show significant differences in terms of success rates across the type of host institution ${ }^{20}$ once models were adjusted, the significantly fewer number of awards probably highlights important differences in the level of systematic support across professional roles and institutions.

In this quantitative analysis, we also found that holding a previous NIHR award makes a difference to success rates ${ }^{20}$ and so the presence or absence of early and active sponsorship may have a long-term impact on academic career development. ${ }^{24}$ Individual careers featuring early awards are much more likely to be found in some professions than others, for a variety of reasons. The availability of early career awards has been longer established in some professions, particularly medicine, than in others. The key predictors of success in an NIHR application were the level and type of programme applied for, holding a previous award and certain characteristic of the institution or region the application was from..$^{25}$ The most important of which was association of the institution with a medical school.

The study by Trusson $e t a l^{14}$ (also in the UK) is one of few in the literature to have compared the experiences of doctors and their non-medical professional counterparts, and no study before this one has systematically sought accounts from research managers in a position to make within-institution comparisons. The comparative findings are consistent: a healthy research culture and institutional support are important factors in success across disciplines. However, while many institutions provide these for their medical staff, non-medical health professionals are much more likely to have to rely on chance encounters with individual role models and senior managers to provide the encouragement and support that they need. The international evidence from studies of separate disciplines is entirely consistent with this picture.

\section{Perceptions of short-termism}

The potential to develop a longer-term career as an independent researcher was a concern to some. The increased 
dependence of research on a mostly non-tenured workforce is a growing concern in Higher Education Institutions (HEIs) ${ }^{26}$ and is a factor contributing to the reduced attractiveness of a clinical research career. ${ }^{27}$ Longer term support for research activities postaward was seen as a major barrier to developing a research career for nurses and AHPs, in particular, the highest proportion of which are women; couppled with the perception of a career 'cliff edge' (ie, the potential for an abrupt end), these barriers have been identified previously in regards to this group. ${ }^{28}$ The NIHR Academy's new bridging schemes will be key to supporting career progression and need to be widely promoted if career pathways are to be seen as attractive.

Short-termism has been identified as an almost universal problem in the literature, afflicting non-medical research careers most of all. ${ }^{81113}$ Internationally, a dependable career pathway is lacking for researchers from all discipline backgrounds, but the lack of career opportunities for nurses, midwives and AHPs is particularly striking and has been recognised in Australia and the UK as requiring a coordinated and wide-ranging response. ${ }^{129}$

\section{Perceptions of gender inequality}

A particular concern of the review was to establish any inequalities by gender within the NIHR training programmes. The quantitative analysis showed that since the programmes began, more women have applied for and held personal awards than men at roughly a 60:40 ratio, and overall success rates are similar between women $(21 \%)$ and men $(22 \%) \cdot{ }^{30}$ While the gender split changes that approximately two times as many men as women hold awards at Chair level, nearly two times as many men as women applied for these awards and success rates again were not significantly different by gender. This suggests that the NIHR awarding processes are not gender biased once women apply, and that women academics and researchers must be supported within their institutions to make more applications. However, focussing only on this aspect reduces scrutiny on gender inequalities embedded within organisational structures, policies, funding requirements and expectations of award holders. ${ }^{31}$ It has been argued that the concept of academic excellence is itself gendered, valuing the 'masculine' ideal of the individual star above collaboration within research. ${ }^{32} 33$ This may influence who is in the pool of those supported to apply for personal award. The NIHR Academy has the opportunity to ensure that they do not sustain stereotyped perceptions of research excellence and champion more varied models of excellence within research careers.

Some potential structural inequalities were identified in the interview study. For example, women tend to be relatively senior in their career before embarking on research leadership, a time which often coincides with childbearing and family caring responsibilities and may not be compatible with traditional ideas of the 'career-focused' research leader. The NIHR Academy has removed 'years since PhD' as an eligibility criteria for post-doc awards, and there are several examples of people starting a $\mathrm{PhD}$ later in their clinical career, especially through the Integrated Clinical Academic programme, but small numbers may mean that individuals are not visible. The challenge of satisfactorily combining family responsibilities with a clinical/research/teaching workload should not be underestimated $^{34}$ Senior clinical academic careers, in particular, are highly competitive, explicitly and implicitly privileging individuals with maximum flexibility of both time (a long-hour culture, short deadlines) and location (moving jobs, international collaborations).

Ongoing issues around the compatibility or otherwise of research and clinical practice in some professions predominantly staffed by women and this will affect all the career stages of some potential applicants. Unpredictability of work demands is often integral to a clinical academic role. If out-of-work demands are nonnegotiable (such as childcare), then unpredictability is unmanageable. Importantly, therefore, the 'gender gap' in senior applications may better reflect a research infrastructure historically developed to support the career trajectories of men who work full time rather than a lack of self-confidence or motivation in women. ${ }^{35}$ The existing provision of the NIHR part-time fellowships is important to creating a more inclusive research culture, as long as these facilitate longer term research careers in the same way as do full-time awards; something that could be evaluated.

The influence of gender on career progression in medicine has been widely studied, particularly in North America. ${ }^{89}$ Bias based on gender and other protected characteristics was reported as widespread by interviewees in the UK study by Finn et al. ${ }^{8}$ The Canadian study of award success by Burns $e t a l^{23}$ accepted gender bias as a likely contributory factor to differential success rates. The emerging literature on the research careers of nurses, midwives and AHPs has contributed additional evidence of problems most likely to affect women, such as job insecurity and work-life balance. ${ }^{11131421}$ However, the intertwining of individual, professional and institutional factors discussed in the sections above clearly indicate that the gender differences in research careers observed internationally are almost certainly attributable to factors over and beyond the biases of individual decision-makers, important though the latter can potentially be. Australia and the UK are now both acknowledging the need for much more comprehensive strategies to address the wider problem. ${ }^{129}$

\section{Future directions for the NIHR Academy}

Since the 'Ten years on report' was published and this work undertaken, the NIHR TCC has been brought under the umbrella of the new NIHR Academy, and a number of changes have already been made. ${ }^{36}$ The programmes have been simplified (see figure 1), but more importantly, increased flexibility and extended eligibility have been introduced in order to attract groups that had previously struggled to apply. NIHR is aware of the lack of support from hosting institutions, and although more 
work is needed a letter in summer 2019 from the NIHR with the MRC and Wellcome to institutions hosting fellowships set out the requirements to support awardees, with the possibility of sanctions should this support not be forthcoming. ${ }^{37}$ The NIHR Academy is building capacity in research where evidence to underpin good practice is most limited, including some awards being linked to NIHR Strategic Priorities. In terms of gender equality, work across funders to further understand the relative under-representation of women in academic medicine, especially at senior levels, has been commissioned. ${ }^{36} \mathrm{~A}$ maximum of two nominations are permitted per HEI for the NIHR Research Professorships and where two nominees are put forward, at least one of these must be woman. ${ }^{38}$ Since 2011 , the NIHR has championed implementation of the Athena Swan Charter for women in science. Securing the Silver level of this award has until recently been a condition of funding for organisations wishing to host IAT awards. This intervention has been very influential in changing the landscape in respect to gender equality and has led to the greater embedding of equality practice. It is not known whether other national gender equality initiative such as SAGE in Australia have had a similar impact.

Developing a positive research culture and ensuring greater support for the career development of researchers have gained more prominence in the last year with several initiatives supported by cross-sector stakeholder groups and the government's Research and Development Roadmap highlighting the need for action to be taken. ${ }^{20-22}$

Everyone interviewed, without exception, considered the NIHR training awards to be highly prestigious with the potential to change the career trajectories and lives of award holders. For this reason, and to meet the growing need for evidence-based health and care across a wider range of research areas, greater consistency of research support infrastructure is an important future goal for national clinical research training programmes. The following suggestions are made with this goal in mind:

1. Actively promote the development of healthy research cultures within host institutions by

a. Setting clear expectations for, and provides robust oversight of, support, sponsorship and mentoring practices. National clinical research training programmes could make more effective use of the financial value and prestige of its schemes to some institutions to leverage improvements, especially for under-represented groups; an approach taken by the NIHR already in terms of Athena Swan requirements.

b. Considering how to encourage and reward longer term support for personal awardees, for example, creation of postfellowship development plans.

c. Working with low research intensity institutions to consider how to better embed research outside of universities, for example, pairing organisations with a successful organisation in a mentoring relationship.

2. Conduct further focused work on how to address the additional challenges facing nursing and AHP staff wishing to develop research careers and promote the use of bridging schemes to help with career progression.

3. Further initiatives to improve equality of opportunity and outcome in academic medicine, including promotion of a more diverse range of research role models, for example, those working in flexible or part-time roles or from ethnic minority backgrounds bearing in mind the influence of race on academic career development. ${ }^{39}$

4. Challenging perceptions of an unequal 'playing field' within schemes, where evidence does not support them. For the NIHR, there is a need to raise awareness of the equal success rate of women and men once they apply for awards, transparency about the broad range of award panels constituents and distribution of awards geographically and across a range of host organisations, not just those in the UK 'Golden Triangle'.

5. Conduct regular review ${ }^{20}$ and qualitative assessments to identify any progress and impact of the changes to the programmes and actions to improve transparency. This study has some acknowledged limitations. The interviews, conducted in mid-2017, present a snapshot of the views and experiences of 22 individuals, from a limited range of professions covered by the schemes, all of whom had been successful at least once in obtaining an NIHR personal award. Although we attempted to interview as a diverse range of people as possible within the constraints of the project we are aware that some views may not have been captured or all the important questions addressed. In particular, work to understand how to 'level the playing field' for AHPs and nurses in particular is a priority. Finally, our sample was not diverse in terms of race (only one interviewee self-identified as BAME) and no analysis by protected characteristic other than sex was conducted as part of the quantitative review as the NIHR does not hold data on protected characteristics at an individual level. Further research would be beneficial to help explore experiences across a more diverse range of individuals and the experiences of those who remain unsuccessful applicants.

\section{CONCLUSIONS}

Our interviews identified that personal awardees and managers perceived many strengths of the NIHR training programmes. These strengths include 'transformative' opportunities for developing individual careers and in delivering research capacity to address evolving challenges within the NHS. Our analysis identified some areas for improvement, which could enhance the ability of the NIHR Academy, and other similar national training schemes, to attract and develop the best research professionals from a diverse range of backgrounds, professions, 
specialities and institutions. The Academy has already substantially changed its training programmes to address many of these areas; however, perceptions of inequality may still persist, especially in AHPs and nurses and those not within the UK 'Golden Triangle' or Russell Group medical schools. Further work is needed to overcome such perceptions as they may be acting as a barrier to applicants from certain groups that the NIHR Academy most wanted to attract and develop.

Finally, while national research training programmes can make changes to develop and continuously improve their own processes and awards, for their goals to become fully realised, the organisations within which clinical research takes place must be full partners in, and coleaders of, these changes. A healthy research environment is crucial for the success of clinical academic training schemes to deliver and sustain the research workforce needed to address our future global health challenges.

Contributors PB contributed to the development of the interview schedule, conducted all the interviews, conducted the framework analysis and produced the findings. She made significant contributions to the final report and this paper. LDB contributed to the design of the study and the development of the interview schedule, supervised the qualitative research, and made significant contributions to the final report and this paper, and is responsible for the overall content as the guarantor. CM contributed to the development of the interview schedule, co-ordinated the recruitment of participants and contributed to drafts of the final report and this paper. PT contributed to the design of the study, drafts of the final report and this paper. LAC contributed to the design of the study, drafts of the final report and this paper. MRM contributed to the design of the study, co-ordinated the recruitment of participants and contributed to drafts of the final report and this paper. JH oversaw the whole review, contributed to the design of the qualitative study and the development of the interview schedule, made significant contributions to the final report and this paper.

Funding This work was supported by the NIHR Academy and the University of Leeds, School of Medicine.

Competing interests CM, PT and LAC are employed by the Leeds Teaching Hospitals Trust, JH was a member then Chair of a selection panel for NIHR Academy Fellowships between 2006 and 2014.

Patient and public involvement Patients and/or the public were not involved in the design, or conduct, or reporting, or dissemination plans of this research.

Patient consent for publication Not applicable.

Ethics approval This study involves human participants and was approved by School of Medicine Research Ethics Committee at the University of Leeds ID: MREC16-101. Participants gave informed consent to participate in the study before taking part.

Provenance and peer review Not commissioned; externally peer reviewed.

Data availability statement Data are available upon reasonable request. All requests for data will be considered by the author team. Available data are anonymised tabulated summaries of the interview.

Open access This is an open access article distributed in accordance with the Creative Commons Attribution Non Commercial (CC BY-NC 4.0) license, which permits others to distribute, remix, adapt, build upon this work noncommercially, and license their derivative works on different terms, provided the original work is properly cited, appropriate credit is given, any changes made indicated, and the use is non-commercial. See: http://creativecommons.org/ licenses/by-nc/4.0/.

\section{ORCID iDs}

Louise D Bryant http://orcid.org/0000-0002-1972-7395

Caroline Magee http://orcid.org/0000-0003-0352-7142

Lisa Ann Cotterill http://orcid.org/0000-0002-4083-3483

Matthew R Mulvey http://orcid.org/0000-0002-6357-3848
REFERENCES

1 Eley DS, O Leary SP, Young A, et al. Is Australia's clinician scientist capacity appropriate for addressing the next pandemic? Aust Health Rev 2021;45:308-10.

2 Medical Schools Council. Survey of medical clinical academic staffing levels 2017: a report by the medical schools Council. London: Medical Schools Council, 2017.

3 Dos Santos Rocha A, Combescure C, Negro F. The MD-PhD program in Geneva: a 10-year analysis of graduate demographics and outcomes. BMC Med Educ 2020;20:425.

4 Strong MJ, Busing N, Goosney DL, et al. The rising challenge of training physician-scientists: recommendations from a Canadian national consensus conference. Acad Med 2018;93:172-8.

5 Weggemans MM, Friesen F, Kluijtmans M, et al. Critical gaps in understanding the clinician-scientist workforce: results of an international expert meeting. Acad Med 2019;94:1448-54.

6 Brown JVE, Crampton PES, Finn GM, et al. From the sticky floor to the glass ceiling and everything in between: protocol for a systematic review of barriers and facilitators to clinical academic careers and interventions to address these, with a focus on gender inequality. Syst Rev 2020;9:26.

7 Chakraverty D, Jeffe DB, Dabney KP, et al. Exploring reasons that U.S. MD-PHD students enter and leave their DUAL-DEGREE programs. Int J Dr Stud 2020;15:461-83.

8 Finn GU, Raine EP, Buchanan G. From the sticky floor to the glass ceiling and everything in between: a systematic review and qualitative study focusing on inequalities in clinical academic careers, 2021.

9 Vassie C, Smith S, Leedham-Green K. Factors impacting on retention, success and equitable participation in clinical academic careers: a scoping review and meta-thematic synthesis. BMJ Open 2020;10:e033480.

10 Yoon S, Koh W-P, Ong MEH, et al. Factors influencing career progress for early stage clinician-scientists in emerging Asian academic medical centres: a qualitative study in Singapore. BMJ Open 2018;8:e020398.

11 Brandenberg CW E. "There hasn't been a career structure to step into": a qualitative study on perceptions of allied health clinician researcher careers. Research Square 2021.

12 Cowley A, Diver C, Edgley A, et al. Capitalising on the transformational opportunities of early clinical academic career training for nurses, midwives and allied health professionals. BMC Med Educ 2020;20:418.

13 Matus J, Walker A, Mickan S. Research capacity building frameworks for allied health professionals - a systematic review. BMC Health Serv Res 2018;18:716.

14 Trusson D, Rowley E, Barratt J. Multimethods study comparing the experiences of medical clinical academics with nurses, midwives and allied health professionals pursuing a clinical academic career. BMJ Open 2021;11:e043270.

15 National Institute for Health Research. Ten years on: adapting and evolving to new challenges in developing tomorrow's health research leaders. Leeds: NIHR TCC, 2017.

16 National Institute for Health Research. Strategic review of training. Available: https://www.nihr.ac.uk/our-research-community/NIHRacademy/about-the-nihr-academy/strategic-review-of-training.htm [Accessed 02/11018].

17 Spencer L, Ritchie J. Qualitative data analysis for applied policy research. Routledge: Analyzing qualitative data, 2002: 187-208.

18 Pope C, Ziebland S, Mays N. Analysing qualitative data. British Medical journal 2000;320:114-6.

19 QSR International Pty Ltd. NVivo qualitative data analysis software [program]. Version 10 version, 2012.

20 Mulvey MR, West R, Cotterill LA, et al. Ten years of NIHR research training: who got an NIHR Academy Award? BMJ Open Accepted 2021.

21 Avery M, Westwood G, Richardson A. Enablers and barriers to progressing a clinical academic career in nursing, midwifery and allied health professions: a cross-sectional survey. J Clin Nurs 2021;6. doi:10.1111/jocn.15673. [Epub ahead of print: 28 Jan 2021].

22 Clough S, Fenton J, Harris-Joseph H, et al. What impact has the NIHR academic clinical fellowship (ACF) scheme had on clinical academic careers in England over the last 10 years? A retrospective study. BMJ Open 2017;7:e015722.

23 Burns KEA, Straus SE, Liu K, et al. Gender differences in grant and personnel award funding rates at the Canadian Institutes of health research based on research content area: a retrospective analysis. PLoS Med 2019;16:e1002935.

24 Gottlieb AS, Travis EL. Rationale and models for career advancement sponsorship in academic medicine: the time is here; the time is now. Acad Med 2018;93:1620-3. 
25 Cotterill L, Hanley N, Hewison J. Ten years on: Adaptcing and evolving to new challenges in developing tomorrow's health research leaders. Lodon: Centre NTC, 2017.

26 Crawford T, Germov J. Using workforce strategy to address academic casualisation: a university of Newcastle case study. J High Educ Policy Manag 2015;37:534-44.

27 Medical Research Council. UK-wide survey of clinical and health research fellowships, 2017. Available: https://mrc.ukri.org/ publications/browse/clinical-and-health-research-fellowships-survey2017/

28 Trusson D, Rowley E, Bramley L. A mixed-methods study of challenges and benefits of clinical academic careers for nurses, midwives and allied health professionals. BMJ Open 2019;9:e030595.

29 Jones D, Keenan A-M. The rise and rise of NMAHPs in UK clinical research. Future Healthc J 2021;8:e195-7.

30 Moss-Racusin CA, Dovidio JF, Brescoll VL, et al. Science faculty's subtle gender biases favor male students. PNAS 2012;109:16474-9.

31 Bryant LD, Burkinshaw P, House AO, et al. Good practice or positive action? Using $Q$ methodology to identify competing views on improving gender equality in academic medicine. BMJ Open 2017;7:e015973.
32 Burkinshaw P, Cahill J, Ford J. Empirical evidence illuminating gendered regimes in UK higher education: developing a new conceptual framework. Educ Sci 2018;8:81.

33 Van den Brink M. Scouting for talent: appointment practices of women professors in academic medicine. Soc Sci Med 2011;72:2033-40.

34 Ranieri V, Barratt H, Fulop N, et al. Factors that influence career progression among postdoctoral clinical academics: a scoping review of the literature. BMJ Open 2016;6:e013523.

35 Edmunds LD, Ovseiko PV, Shepperd S, et al. Why do women choose or reject careers in academic medicine? A narrative review of empirical evidence. The Lancet 2016;388:2948-58.

36 National Institute for Health Research. NIHR strategic review of trainining implementation plans, 2018.

37 Nature Editorial. Younger scientists need better support. Nature 2019;572:563.

38 National Institute for Health Research. NIHR research Professorships. NIHR Academy, 2018.

39 Wanelik KM, Griffin JS, Head M. Breaking barriers: the effect of protected characteristics and their intersectionality on career transition in academics 2019:593905. 